\title{
Inverse of an Arbitrary Vandermonde Matrix
}

\section{J. López-Bonilla ${ }^{1}$, S. Vidal-Beltrán ${ }^{1}$, J.M. Rivera-Rebolledo ${ }^{2}$}

${ }^{1}$ ESIME-Zacatenco, Instituto Politécnico Nacional (IPN), Anexo Edif. 3, Col. Lindavista CP 07738, México DF, E-mail: jlopezb@ipn.mx

${ }^{2}$ Depto. Física, ESFM-IPN, Edif. 9, Col. Lindavista CP 07738, México DF

Abstract. It is shown a general formula to invert any type Vandermonde matrix.

The Vandermonde [1-3] matrices have the structure:

$$
\left(\begin{array}{ll}
1 & x_{1} \\
1 & x_{2}
\end{array}\right),\left(\begin{array}{lll}
1 & x_{1} & x_{1}^{2} \\
1 & x_{2} & x_{2}^{2} \\
1 & x_{3} & x_{3}^{2}
\end{array}\right),\left(\begin{array}{llll}
1 & x_{1} & x_{1}^{2} & x_{1}^{3} \\
1 & x_{2} & x_{2}^{2} & x_{2}^{3} \\
1 & x_{3} & x_{3}^{2} & x_{3}^{3} \\
1 & x_{4} & x_{4}^{2} & x_{4}^{3}
\end{array}\right), \cdots
$$

and are useful in the Lagrange interpolation [4]. Here it is exhibited a general expression to construct the inverse of a matrix of the type (1); in fact, let

$$
V=\left(\begin{array}{ccccc}
1 & x_{1} & x_{1}^{2} & \cdots & x_{1}^{n-1} \\
1 & x_{2} & x_{2}^{2} & \cdots & x_{2}^{n-1} \\
\vdots & \vdots & \vdots & \vdots & \vdots \\
1 & x_{n} & x_{n}^{2} & \cdots & x_{n}^{n-1}
\end{array}\right), \quad n=2,3, \cdots
$$

from which it is well known [2] that its determinant is not zero if the $x_{j}$ are different among them, then under this last assumption there exists $V_{\sim}^{-1}=\left(A_{r k}\right)$, and for their elements it is presented the following formula

$$
A_{r k}=\frac{(-1)^{n+r}}{B_{k}} C_{r k},
$$

where: 


$$
\begin{aligned}
& B_{k}=\prod_{\substack{j=1 \\
j \neq k}}^{n}\left(x_{k}-x_{j}\right), \quad C_{n k}=1, \quad k=1,2, \cdots, n \\
& C_{r k}=\sum_{i_{1}, \cdots, i_{c}} x_{i_{1}} x_{i_{2}} \cdots x_{i_{c}}, \quad \begin{array}{l}
c=n-r, \quad r=1, \cdots, n-1, \\
i_{j} \neq k, \quad j=1, \cdots, c,
\end{array} \\
& i_{1}=n, n-1, \cdots, c, \quad i_{2}=i_{1}-1, \cdots, 1 \cdots, \quad i_{c}=i_{c-1}-1, \cdots, 1,
\end{aligned}
$$

which for $n=2,3,4, \ldots$ leads to the inverse matrices:

$$
\begin{aligned}
& \frac{1}{B_{2}}\left(\begin{array}{cc}
x_{2} & -x_{1} \\
-1 & 1
\end{array}\right), \quad B_{2}=-B_{1}=x_{2}-x_{1}, \\
& \left(\begin{array}{ccc}
\frac{x_{2} x_{3}}{B_{1}} & \frac{x_{1} x_{3}}{B_{2}} & \frac{x_{1} x_{2}}{B_{3}} \\
-\frac{x_{2}+x_{3}}{B_{1}} & -\frac{x_{1}+x_{3}}{B_{2}} & -\frac{x_{1}+x_{2}}{B_{3}} \\
\frac{1}{B_{1}} & \frac{1}{B_{2}} & \frac{1}{B_{3}}
\end{array}\right), \begin{array}{l}
B_{1}=\left(x_{1}-x_{2}\right)\left(x_{1}-x_{3}\right), \\
B_{2}=\left(x_{2}-x_{1}\right)\left(x_{2}-x_{3}\right),(5) \\
B_{3}=\left(x_{3}-x_{2}\right)\left(x_{3}-x_{1}\right),
\end{array} \\
& \left(\begin{array}{cccc}
-\frac{x_{2} x_{3} x_{4}}{B_{1}} & -\frac{x_{1} x_{3} x_{4}}{B_{2}} & \frac{x_{1} x_{2} x_{4}}{B_{3}} & \frac{x_{1} x_{2} x_{3}}{B_{4}} \\
\frac{x_{2} x_{3}+x_{2} x_{4}+x_{3} x_{4}}{B_{1}} & \frac{x_{1} x_{3}+x_{1} x_{4}+x_{3} x_{4}}{B_{2}} & \frac{x_{1} x_{2}+x_{1} x_{4}+x_{2} x_{4}}{B_{3}} & \frac{x_{1} x_{2}+x_{1} x_{3}+x_{2} x_{3}}{B_{4}} \\
-\frac{x_{2}+x_{3}+x_{4}}{B_{1}} & -\frac{x_{1}+x_{3}+x_{4}}{B_{2}} & \frac{x_{1}+x_{2}+x_{4}}{B_{3}} & \frac{x_{1}+x_{2}+x_{3}}{B_{4}} \\
\frac{1}{B_{1}} & \frac{1}{B_{2}} & \frac{1}{B_{3}} & \frac{1}{B_{4}}
\end{array}\right.
\end{aligned}
$$

with:

$$
\begin{array}{ll}
B_{1}=\left(x_{1}-x_{2}\right)\left(x_{1}-x_{3}\right)\left(x_{1}-x_{4}\right), & B_{2}=\left(x_{2}-x_{1}\right)\left(x_{2}-x_{3}\right)\left(x_{2}-x_{4}\right), \\
B_{3}=\left(x_{3}-x_{1}\right)\left(x_{3}-x_{2}\right)\left(x_{3}-x_{4}\right), & B_{4}=\left(x_{4}-x_{1}\right)\left(x_{4}-x_{2}\right)\left(x_{4}-x_{3}\right),
\end{array}
$$

which in turn give the identity matrix after multiplication by (1).

Turner [5], see its relations (6) and (8), showed that $V_{n \times n}^{-1}$ is the product of two triangular matrices, which can be illustrated with the inverse matrices (5):

$$
V_{2 \times 2}^{-1}=\left(\begin{array}{cc}
1 & -x_{1} \\
0 & 1
\end{array}\right)\left(\begin{array}{cc}
1 & 0 \\
\frac{1}{B_{1}} & \frac{1}{B_{2}}
\end{array}\right),
$$




$$
\begin{gathered}
V_{3 \times 3}^{-1}=\left(\begin{array}{ccc}
1 & -x_{1} & x_{1} x_{2} \\
0 & 1 & -\left(x_{1}+x_{2}\right) \\
0 & 0 & 1
\end{array}\right)\left(\begin{array}{ccc}
1 & 0 & 0 \\
\frac{1}{x_{1}-x_{2}} & \frac{1}{x_{2}-x_{1}} & 0 \\
\frac{1}{B_{1}} & \frac{1}{B_{2}} & \frac{1}{B_{3}}
\end{array}\right), \\
V_{4 \times 4}^{-1}=\left(\begin{array}{cccc}
1 & -x_{1} & x_{1} x_{2} & -x_{1} x_{2} x_{3} \\
0 & 1 & -\left(x_{1}+x_{2}\right) & x_{1} x_{2}+x_{2} x_{3}+x_{1} x_{3} \\
0 & 0 & 1 & -\left(x_{1}+x_{2}+x_{3}\right) \\
0 & 0 & 0 & 1
\end{array}\right) \cdot T,
\end{gathered}
$$

with:

$$
T=\left(\begin{array}{cccc}
1 & 0 & 0 & 0 \\
\frac{1}{x_{1}-x_{2}} & \frac{1}{x_{2}-x_{1}} & 0 & 0 \\
\frac{1}{\left(x_{1}-x_{2}\right)\left(x_{1}-x_{3}\right)} & \frac{1}{\left(x_{2}-x_{1}\right)\left(x_{2}-x_{3}\right)} & \frac{1}{\left(x_{3}-x_{1}\right)\left(x_{3}-x_{2}\right)} & 0 \\
\frac{1}{B_{1}} & \frac{1}{B_{2}} & \frac{1}{B_{3}} & \frac{1}{B_{4}}
\end{array}\right) .
$$

The expressions (3) and (4) are easy to manage with MAPLE or by means of any Symbolic Program, with an immediate application to polynomials interpolation problems [4].

\section{REFERENCES}

[1] C. Lanczos, Applied analysis, Dover, New York (1988)

[2] F.D. Parker, Am. Math. Monthly $\underline{71}$, No. 4 (1964) 410 - 411

[3] J.H. Wilkinson, The algebraic eigenvalue problem, Clarendon Press, Oxford (1965)

[4] L.R. Turner, Lewis Research Center-NASA, Cleveland, Ohio (1966)

[5] R.A. Horn and Ch.R. Johnson, Matrix analysis, Cambridge University Press (1990) 